\title{
A new extragradient algorithm with adaptive step-size for solving split equilibrium problems
}

\author{
Yusuf I. Suleiman ${ }^{1}$ (D), Poom Kumam ${ }^{2,3^{*}}$ (D) Habib ur Rehman ${ }^{2}$ (D) and Wiyada Kumam ${ }^{4 *}$ (1)
}

\section{"Correspondence:}

poom.kum@kmutt.ac.th;

wiyada.kum@rmutt.ac.th

${ }^{2}$ Center of Excellence in Theoretical

and Computational Science

(TaCS-CoE) \& KMUTTFixed Point

Research Laboratory, Room SCL 802 Fixed Point Laboratory, Science

Laboratory Building, Departments of Mathematics, Faculty of Science,

King Mongkut's University of

Technology Thonburi (KMUTT), 126

Pracha-Uthit Road, Bang Mod,

Thung Khru, Bangkok 10140,

Thailand

${ }^{4}$ Applied Mathematics for Science and Engineering Research Unit (AMSERU), Program in Applied Statistics, Department of

Mathematics and Computer Science, Faculty of Science and Technology, Rajamangala University of Technology Thanyaburi,

Thanyaburi, Pathumthani 12110,

Thailand

Full list of author information is available at the end of the article

\begin{abstract}
He (J. Inequal. Appl. 2012:Article ID 162 2012) introduced the proximal point CQ algorithm (PPCQ) for solving the split equilibrium problem (SEP). However, the PPCQ converges weakly to a solution of the SEP and is restricted to monotone bifunctions. In addition, the step-size used in the PPCQ is a fixed constant $\mu$ in the interval $\left(0, \frac{1}{\|A\|^{2}}\right)$. This often leads to excessive numerical computation in each iteration, which may affect the applicability of the PPCQ. In order to overcome these intrinsic drawbacks, we propose a robust step-size $\left\{\mu_{n}\right\}_{n=1}^{\infty}$ which does not require computation of $\|A\|$ and apply the adaptive step-size rule on $\left\{\mu_{n}\right\}_{n=1}^{\infty}$ in such a way that it adjusts itself in accordance with the movement of associated components of the algorithm in each iteration. Then, we introduce a self-adaptive extragradient-CQ algorithm (SECQ) for solving the SEP and prove that our proposed SECQ converges strongly to a solution of the SEP with more general pseudomonotone equilibrium bifunctions. Finally, we present a preliminary numerical test to demonstrate that our SECQ outperforms the PPCQ.
\end{abstract}

MSC: 47H05; 47H09; 49M37;65K10

Keywords: Split equilibrium problems; Extragradient algorithm; Self-adaptive step-sizes; Pseudomonotone equilibrium problems

\section{Introduction}

The equilibrium problem (EP) associated with a bifunction $f: C \times C \rightarrow \mathbb{R}$ and a nonempty subset $C$ of a real Hilbert space $\mathbb{H}$ consists of finding a vector $x^{*} \in C$ such that

$$
f\left(x^{*}, y\right) \geq 0, \quad \forall y \in C .
$$

It is well known that the mathematical basis for the EP pre-dates the works of Ky Fan [10]. However, due to his dedication to the subject, the EP is often called the Ky Fan inequality. The EP is an incredibly important powerful tool that unifies a number of useful and elegant nonlinear problems. In recent days, the EP is one of the major nonlinear methods that has provided significant success in modeling several real world problems (see, e.g., [1, 2, 8, 9, $13,14,18,23,24])$.

(c) The Author(s) 2021. This article is licensed under a Creative Commons Attribution 4.0 International License, which permits use, sharing, adaptation, distribution and reproduction in any medium or format, as long as you give appropriate credit to the original author(s) and the source, provide a link to the Creative Commons licence, and indicate if changes were made. The images or other third party material in this article are included in the article's Creative Commons licence, unless indicated otherwise in a credit line to the material. If material is not included in the article's Creative Commons licence and your intended use is not permitted by statutory regulation or exceeds the permitted use, you will need to obtain permission directly from the copyright holder. To view a copy of this licence, visit http://creativecommons.org/licenses/by/4.0/. 
The set of solutions of EP is denoted by $\operatorname{SOL}(f, C)$. To solve the EP, it is common to use the proximal point method proposed by Combettes and Hirstoaga [8]: given $x_{n} \in C$, as a current iterate, the next iterate $x_{n+1}$ solves the following problem:

$$
\text { find } x \in C \text { such that } f(x, y)+\frac{1}{r_{n}}\left\langle y-x, x-x_{n}\right\rangle \geq 0, \quad \forall y \in C,\left\{r_{n}\right\}_{n=1}^{\infty} \subset(0, \infty) \text {. }
$$

In 2008, Tran et al. [23] (see also Dang [9]) submitted that a large number of important real-world problems can be reformulated as pseudomonotone bifunctions. A well-known example is the Nash-Cournot oligopolistic electricity market model. Unfortunately, the traditional proximal point method (2) does not converge if $f$ is pseudomonotone (see, e.g., [21, Example 2.1]). Hence, Tran et al. [23] introduced the following proximal-extragradient algorithm for solving (1) when $f$ is pseudomonotone.

$$
\left\{\begin{array}{l}
\text { select arbitrary } \quad x_{1} \in C, \\
y_{n}=\operatorname{argmin}_{y \in C}\left\{f\left(x_{n}, y\right)+\frac{1}{2 \rho_{n}}\left\|y-x_{n}\right\|^{2}\right\}, \\
x_{n+1}=\operatorname{argmin}_{y \in C}\left\{f\left(y_{n}, y\right)+\frac{1}{2 \rho_{n}}\left\|y-x_{n}\right\|^{2}\right\}, \quad n \in \mathbb{N} .
\end{array}\right.
$$

It is worthy to mention that, unlike the proximal point method (2), the extragradient algorithm (3) falls within the applicable scope of standard matlab optimization toolbox. So, in order to implement algorithm (3), one only needs to solve a pair of strongly convex programs via matlab optimization toolbox.

On the other hand, the study of classical split feasibility problem (SFP) is pioneered by Censor and Elfving [5]. It provides effective tools for obtaining the existence of solutions of constrained and inverse problems arising in optimization, engineering, medical sciences, and most notably in image reconstruction, signal processing, and phase retrieval (see, e.g., $[3,4,6])$. The SFP is to find a vector

$$
x^{*} \in C \text { such that } A\left(x^{*}\right) \in Q
$$

where $C$ and $Q$ are given nonempty, closed, and convex subsets of real Hilbert spaces $\mathrm{H}_{1}$ and $\mathrm{H}_{2}$, respectively, and $A: \mathrm{H}_{1} \rightarrow \mathrm{H}_{2}$ is a bounded linear operator. Bryne [3] imposed the restrictive condition $\left\{\gamma_{n}\right\}_{n=1}^{\infty} \subset\left(0, \frac{2}{\|A\|^{2}}\right)$ on the CQ-method for solving (4):

$$
x_{1} \in C, \quad x_{n+1}:=\mathrm{P}_{C}\left(x_{n}-\gamma_{n} A^{*}\left(I-\mathrm{P}_{\mathrm{Q}}\right) A x_{n}\right), \quad n \in \mathbb{N},
$$

where $\mathrm{P}_{C}$ and $\mathrm{P}_{Q}$ stand for the metric projection onto $C$ and $Q$, respectively, and $A^{*}$ denotes the adjoint operator of $A$ from $\mathrm{H}_{2}$ to $\mathrm{H}_{1}$. The concept of self-adaptive step-size for solving (4) was introduced by Yang [26] and developed by Lopez et al. [15] in order to dispense with the restrictive condition $\left\{\gamma_{n}\right\}_{n=1}^{\infty} \subset\left(0, \frac{2}{\|A\|^{2}}\right)$. In general, self-adaptive algorithms operate under the assumption that future events (inputs) are uncertain so they are characterized by step-sizes that continuously monitor themselves, gather data, analyze data, and adapt when their requirements fail due to unexpected changes in their components. Research found out that an ever-growing number of algorithms with adaptive step-sizes for solving nonlinear problems are faster and robust to failure (see, e.g., $[11,19,20,22,27,28]$ ). 
The SFP is a particular case of a more general problem, called the split equilibrium problem (SEP)

$$
\left\{\begin{array}{l}
\text { find } x^{*} \in C \text { such that } f\left(x^{*}, y\right) \geq 0, \quad \forall y \in C \quad \text { and } \\
A\left(x^{*}\right)=u^{*} \in Q \quad \text { solves } \quad g\left(u^{*}, v\right) \geq 0, \quad \forall v \in Q .
\end{array}\right.
$$

Here, $g: Q \times Q \rightarrow \mathbb{R}$ stands for another bifunction on $\mathrm{H}_{2}$. The SEP was briefly introduced by Moudafi [17] in 2011. Perhaps, due to its relevance, the problem was reintroduced a year after by He [12], and the following proximal point-CQ algorithm (PPCQ) for solving SEP (6) was proposed:

$$
\left\{\begin{array}{l}
\text { Select arbitrary: } \quad x_{1} \in C, \quad\left\{\rho_{n}\right\}_{n=1}^{\infty} \subset(0, \infty), \quad \text { and } \quad \mu \in\left(0, \frac{1}{\|A\|^{2}}\right), \\
f\left(y_{n}, y\right)+\frac{1}{\rho_{n}}\left\langle y-y_{n}, y_{n}-x_{n}\right\rangle \geq 0, \quad \forall y \in C, \\
g\left(v_{n}, v\right)+\frac{1}{\rho_{n}}\left\langle v-v_{n}, v_{n}-A y_{n}\right\rangle \geq 0, \quad \forall v \in Q, \\
x_{n+1}=P_{C}\left(y_{n}-\mu A^{*}\left(A y_{n}-v_{n}\right)\right), \quad n \in \mathbb{N} .
\end{array}\right.
$$

It is worth noting that, as a prototype of the proximal point method, the PPCQ may not converge when $f$ and $g$ are pseudomonotone. In addition, the PPCQ converges weakly to a solution of (6) when it is consistent and the step-size $\mu \in\left(0, \frac{1}{\|A\|^{2}}\right)$ depends on $\|A\|$, which, in turn, may lead to excessive numerical computation that may affect the convergence of the PPCQ. The question now becomes: is it possible to develop an extragradient algorithm with an adaptive step-size that converges strongly to a solution of (6) when $f$ and $g$ are pseudomonotone? In answering this question, we present a self-adaptive extragradientCQ algorithm (SECQ) for solving (6) and prove that a sequence generated by our proposed SECQ converges strongly to solutions of (6) when $f$ and $g$ are pseudomonotone. A numerical example is also given to demonstrate the effectiveness of our iterative scheme.

\section{Preliminaries}

Let $\mathbb{H}$ be a real Hilbert space whose inner product and norm are denoted by $\langle\cdot, \cdot\rangle$ and $\|\cdot\|$. Let $C \subseteq \mathbb{H}$ be a nonempty, closed, and convex set, and denote by the symbols $\rightarrow$ and $\longrightarrow$ weak and strong convergence of a sequence $\left\{x_{n}\right\}_{n=1}^{\infty}$.

Definition 2.1 A bifunction $f: C \times C \rightarrow \mathbb{R}$ is said to be

(i) monotone on $C$, if

$$
f(x, y)+f(y, x) \leq 0, \quad \forall x, y \in C
$$

(ii) pseudomonotone on $C$ with respect to $x \in C$, if

$$
f(x, y) \geq 0 \quad \Rightarrow \quad f(y, x) \leq 0, \quad \forall y \in C
$$

(iii) pseudomonotone on $C$ with respect to $\emptyset \neq \Omega \subset C$, if $\forall x^{*} \in \Omega$,

$$
f\left(x^{*}, y\right) \geq 0 \quad \Rightarrow \quad f\left(y, x^{*}\right) \leq 0, \quad \forall y \in C ;
$$


(iv) Lipschitz-type continuous, if there are two positive constants $L_{1}, L_{2}$ such that

$$
f(x, y)+f(y, z) \geq f(x, z)-L_{1}\|x-y\|^{2}-L_{2}\|y-z\|^{2}, \quad \forall x, y, z \in C ;
$$

(v) jointly weakly continuous on $C \times C$ in the sense that, given any $x, y \in C$ and $\left\{x_{n}\right\}_{n=1}^{\infty},\left\{y_{n}\right\}_{n=1}^{\infty} \subset C$ converge weakly to $x$ and $y$, respectively, then

$$
\lim _{n \rightarrow \infty} f\left(x_{n}, y_{n}\right)=f(x, y)
$$

The following conditions will be used in the sequel.

\section{Assumption A}

(A1). $f$ is pseudomonotone with respect to $\operatorname{SOL}(f, C)$;

(A2). $f$ is jointly weakly and Lipschitz-type continuous on $C$ with constants $L_{1}$ and $L_{2}$;

(A3). $f(x, \cdot)$ is convex and subdifferentiable on $C$;

(A4). $\Omega=\{x \in \operatorname{SOL}(f, C)$ such that $A(x) \in \operatorname{SOL}(g, Q)\} \neq \emptyset$.

Lemma 2.2 ([2]) If the bifunction $f$ satisfies conditions $(A 1)-(A 4)$, then $\mathrm{SOL}(f, C)$ is weakly closed and convex.

Recall that a metric projection of $\mathbb{H}$ onto $C$ is the mapping $\mathrm{P}_{C}: \mathbb{H} \rightarrow C$ which assigns to each $x \in \mathbb{H}$ the (nearest) unique point $\mathrm{P}_{C}(x)$ in $C$ satisfying

$$
\left\|x-\mathrm{P}_{C}(x)\right\|=\min \{\|x-y\|: y \in C\}
$$

Lemma 2.3 Given $u \in \mathbb{H}$ and $z \in C$. Then

$$
z=\mathrm{P}_{C}(u) \quad \Longleftrightarrow \quad\langle u-z, z-y\rangle \geq 0, \quad \forall y \in C .
$$

Lemma 2.4 ([16]) Let $\left\{a_{n}\right\}_{n=1}^{\infty}$ be a sequence of real numbers such that there exists a subsequence $\left\{n_{i}\right\}$ of $\{n\}$ such that $a_{n_{i}}<a_{n_{i+1}}$ for all $i \geq 0$. Then there exists an increasing sequence $\left\{m_{k}\right\}_{k=1}^{\infty} \subset \mathbb{N}$ such that $m_{k} \rightarrow \infty$ and the following properties are satisfied by all (sufficiently large) numbers $k \in \mathbb{N}$ :

$$
a_{m_{k}} \leq a_{m_{k+1}} \text { and } a_{k} \leq a_{m_{k+1}} .
$$

In fact, $m_{k}$ is the largest number $n$ in the set $\{1,2, \ldots, k\}$ such that the condition $a_{n} \leq a_{n+1}$ holds.

Lemma 2.5 ([25]) Let $\left\{\gamma_{n}\right\}_{n=1}^{\infty}$ be a sequence in $(0,1)$ and $\left\{\delta_{n}\right\}_{n=1}^{\infty}$ be in $\mathbb{R}$ satisfying $\sum_{n=1}^{\infty} \gamma_{n}=\infty$ and $\limsup _{n \rightarrow \infty} \delta_{n} \leq 0$ or $\sum_{n=1}^{\infty}\left|\gamma_{n} \delta_{n}\right|<\infty$. If $\left\{a_{n}\right\}_{n=1}^{\infty}$ is a sequence of nonnegative real numbers such that $a_{n+1} \leq\left(1-\gamma_{n}\right) a_{n}+\gamma_{n} \delta_{n}, \forall n \geq 0$, then $\lim _{n \rightarrow \infty} a_{n}=0$.

\section{Main results}

The following is our main algorithm for solving (6). 
Algorithm 3.1 (Self-adaptive proximal-extragradient algorithm for SEP)

Initialization: Given initial choice $x_{1}$ and $u$ in $C$. Pick parameters

$\left\{\delta_{n}\right\}_{n=1}^{\infty} \subset[\underline{\delta}, \bar{\delta}] \subset(0,1),\left\{\sigma_{n}\right\}_{n=1}^{\infty} \subset(0,2),\left\{\rho_{n}\right\}_{n=1}^{\infty} \subset[\underline{\rho}, \bar{\rho}]$, and $\left\{r_{n}\right\}_{n=1}^{\infty} \subset[\underline{r}, \bar{r}]$ such that

$$
[\underline{\rho}, \bar{\rho}],[\underline{r}, \bar{r}] \subset\left(0, \min \left\{\frac{1}{2 L_{1}}, \frac{1}{2 L_{2}}\right\}\right), \quad \lim _{n \rightarrow \infty} \delta_{n}=0, \quad \text { and } \quad \sum_{i=1}^{N} \delta_{n}=\infty
$$

Iterative steps: Assume that $x_{n}$ is known for $n \in \mathbb{N}$, then compute the update $x_{n+1}$ according to the following rule.

Step 1: Compute:

$$
y_{n}=\underset{y \in C}{\operatorname{argmin}}\left\{f\left(x_{n}, y\right)+\frac{1}{2 \rho_{n}}\left\|y-x_{n}\right\|^{2}\right\} \quad \text { and } \quad z_{n}=\underset{y \in C}{\operatorname{argmin}}\left\{f\left(y_{n}, y\right)+\frac{1}{2 \rho_{n}}\left\|y-x_{n}\right\|^{2}\right\} .
$$

Step 2: Set $\hat{v_{n}}:=\mathrm{P}_{Q} A\left(z_{n}\right)$.

Step 3: Compute:

$$
v_{n}=\underset{v \in Q}{\operatorname{argmin}}\left\{g\left(\hat{v_{n}}, v\right)+\frac{1}{2 r_{n}}\left\|v-\hat{v_{n}}\right\|^{2}\right\} \quad \text { and } \quad u_{n}=\underset{v \in Q}{\operatorname{argmin}}\left\{g\left(v_{n}, v\right)+\frac{1}{2 r_{n}}\left\|v-\hat{v_{n}}\right\|^{2}\right\} .
$$

Step 4: Set $F\left(z_{n}\right):=\frac{1}{2}\left\|A z_{n}-u_{n}\right\|^{2}$ and $G\left(z_{n}\right):=A^{*}\left(A z_{n}-u_{n}\right)$.

Step 5: Compute

$$
\begin{aligned}
& x_{n+1}=\delta_{n} u+\left(1-\delta_{n}\right) \mathrm{P}_{C}\left(z_{n}-\mu_{n} G\left(z_{n}\right)\right), \quad \text { where } \\
& \mu_{n}= \begin{cases}\sigma_{n} \frac{F\left(z_{n}\right)}{\left\|G\left(z_{n}\right)\right\|^{2}}, & \text { if } G\left(z_{n}\right) \neq 0 ; \\
0, & \text { otherwise. }\end{cases}
\end{aligned}
$$

Stopping criterion: If $x_{n+1}=x_{n}$, then $x_{n}$ is a solution of SEP (6) and the iterative process stops, otherwise, put $n:=n+1$ and go back to Step 1 .

Lemma 3.2 ([1], Lemma 3.1) Let $\mathrm{H}_{1}$ and $\mathrm{H}_{2}$ be real Hilbert spaces. Let $C$ and $Q$ be nonempty, closed, and convex subsets of $\mathrm{H}_{1}$ and $\mathrm{H}_{2}$, respectively. Let $A: \mathrm{H}_{1} \rightarrow \mathrm{H}_{2}$ be a bounded linear operator with adjoint $A^{*}$. Assume that $f: C \times C \rightarrow \mathbb{R}$ satisfies conditions (A1)-(A4). Let $\left\{x_{n}\right\}_{n=1}^{\infty}$ be a sequence generated by Algorithm 3.1. Then, for all $x^{*} \in \operatorname{SOL}(f, C)$, the following statements hold:

(a). $\rho_{n}\left(f\left(x_{n}, y\right)-f\left(x_{n}, y_{n}\right)\right) \geq\left\langle y_{n}-x_{n}, y_{n}-y\right\rangle$ for all $y \in C$;

(b). $\left\|z_{n}-x^{*}\right\|^{2} \leq\left\|x_{n}-x^{*}\right\|^{2}-\left(1-2 \rho_{n} L_{1}\right)\left\|x_{n}-y_{n}\right\|^{2}-\left(1-2 \rho_{n} L_{2}\right)\left\|y_{n}-z_{n}\right\|^{2}$.

The following theorem gives conditions that guarantee strong convergence of Algorithm 3.1.

Theorem 3.3 Let $\mathrm{H}_{1}$ and $\mathrm{H}_{2}$ be real Hilbert spaces. Let $C$ and $Q$ be nonempty, closed, and convex subsets of $\mathrm{H}_{1}$ and $\mathrm{H}_{2}$, respectively. Let $A: \mathrm{H}_{1} \rightarrow \mathrm{H}_{2}$ be a bounded linear operator with adjoint $A^{*}$. Assume that $f: C \times C \rightarrow \mathbb{R}$ and $g: Q \times Q \rightarrow \mathbb{R}$ satisfy conditions (A1)(A4). Let $\left\{x_{n}\right\}_{n=1}^{\infty}$ be any sequence generated by Algorithm 3.1. Then $x_{n} \rightarrow \mathrm{P}_{\Omega}(u)$ under the following conditions: 
(C1). $0<t_{1} \leq \mu_{n} \leq t_{2}$ for some $t_{1}, t_{2} \in \mathbb{R}$ and $\forall n \in \Gamma=\left\{n \geq 1: G\left(z_{n}\right) \neq 0\right\} ;$

(C2). $\liminf _{n \rightarrow \infty}\left(2-\sigma_{n}\right)>0$;

(C3). $\left\langle G z_{n}, z_{n}-x^{*}\right\rangle \geq F\left(z_{n}\right)$ for all $x^{*} \in \Omega$.

Proof Let $x^{*}=P_{\Omega}(u)$ and $\pi_{n}=\mathrm{P}_{C}\left(z_{n}-\mu_{n} G\left(z_{n}\right)\right)$. Then, by using $(C 3)$, we have

$$
\begin{aligned}
\left\|\pi_{n}-x^{*}\right\|^{2} & =\left\|\mathrm{P}_{C}\left(z_{n}-\mu_{n} G\left(z_{n}\right)\right)-\mathrm{P}_{C}\left(x^{*}\right)\right\|^{2} \\
& \leq\left\|z_{n}-\mu_{n} G\left(z_{n}\right)-x^{*}\right\|^{2}=\left\|z_{n}-x^{*}-\mu_{n} G\left(z_{n}\right)\right\|^{2} \\
& \leq\left\|z_{n}-x^{*}\right\|^{2}+\left\|\mu_{n} G\left(z_{n}\right)\right\|^{2}-2 \mu_{n}\left|G\left(z_{n}\right), z_{n}-x^{*}\right\rangle \\
& \leq\left\|z_{n}-x^{*}\right\|^{2}+\mu_{n}^{2}\left\|G\left(z_{n}\right)\right\|^{2}-2 \mu_{n} F\left(z_{n}\right) \\
& =\left\|z_{n}-x^{*}\right\|^{2}+\sigma_{n}^{2} \frac{\left[F\left(z_{n}\right)\right]^{2}}{\left\|G\left(z_{n}\right)\right\|^{4}}\left\|G\left(z_{n}\right)\right\|^{2}-2 \sigma_{n} \frac{F\left(z_{n}\right)}{\left\|G\left(z_{n}\right)\right\|^{2}} F\left(z_{n}\right) \\
& =\left\|z_{n}-x^{*}\right\|^{2}+\sigma_{n}^{2} \frac{\left[F\left(z_{n}\right)\right]^{2}}{\left\|G\left(z_{n}\right)\right\|^{2}}-2 \sigma_{n} \frac{\left[F\left(z_{n}\right)\right]^{2}}{\left\|G\left(z_{n}\right)\right\|^{2} .}
\end{aligned}
$$

Thus

$$
\left\|\pi_{n}-x^{*}\right\|^{2} \leq\left\|z_{n}-x^{*}\right\|^{2}-\sigma_{n}\left(2-\sigma_{n}\right) \frac{\left[F\left(z_{n}\right)\right]^{2}}{\left\|G\left(z_{n}\right)\right\|^{2}} .
$$

This implies

$$
\left\|\pi_{n}-x^{*}\right\|^{2} \leq\left\|z_{n}-x^{*}\right\|^{2} .
$$

Consequently, by Lemma 3.2 and (9), we get

$$
\left\|\pi_{n}-x^{*}\right\|^{2} \leq\left\|z_{n}-x^{*}\right\|^{2} \leq\left\|x_{n}-x^{*}\right\|^{2}
$$

By Algorithm 3.1 and (10), we have

$$
\begin{aligned}
\left\|x_{n+1}-x^{*}\right\| & =\left\|\delta_{n} u+\left(1-\delta_{n}\right) \pi_{n}-x^{*}\right\| \\
& =\left\|\delta_{n}\left(u-x^{*}\right)+\left(1-\delta_{n}\right)\left(\pi_{n}-x^{*}\right)\right\| \\
& \leq \delta_{n}\left\|u-x^{*}\right\|+\left(1-\delta_{n}\right)\left\|\pi_{n}-x^{*}\right\| \\
& \leq \delta_{n}\left\|u-x^{*}\right\|+\left(1-\delta_{n}\right)\left\|x_{n}-x^{*}\right\| \\
& \leq \max \left\{\left\|u-x^{*}\right\|,\left\|x_{n}-x^{*}\right\|\right\} \\
& \vdots \\
& \leq \max \left\{\left\|u-x^{*}\right\|,\left\|x_{1}-x^{*}\right\|\right\} .
\end{aligned}
$$

Hence $\left\{x_{n}\right\}_{n=1}^{\infty}$ is bounded. Then, from (10), we deduce that $\left\{\pi_{n}\right\}_{n=1}^{\infty}$ and $\left\{z_{n}\right\}_{n=1}^{\infty}$ are bounded. By using Algorithm 3.1 and subdifferential inequality

$$
\|x+y\|^{2} \leq\|x\|^{2}+2\langle y, x+y\rangle, \quad \forall x, y \in \mathbb{H},
$$


we obtain

$$
\left\|x_{n+1}-x^{*}\right\|^{2} \leq\left(1-\delta_{n}\right)\left\|\pi_{n}-x^{*}\right\|^{2}+2 \delta_{n}\left\langle u-x^{*}, x_{n+1}-x^{*}\right\rangle .
$$

Thus, by Lemma 3.2, (10), and (11), we have

$$
\begin{aligned}
\left\|x_{n+1}-x^{*}\right\|^{2} \leq & \left(1-\delta_{n}\right)\left\|x_{n}-x^{*}\right\|^{2}-\left(1-\delta_{n}\right)\left(\left(1-2 \rho_{n} L_{1}\right)\left\|x_{n}-y_{n}\right\|^{2}\right. \\
& \left.+\left(1-2 \rho_{n} L_{2}\right)\left\|y_{n}-z_{n}\right\|^{2}\right)+2 \delta_{n}\left\langle u-x^{*}, x_{n+1}-x^{*}\right\rangle .
\end{aligned}
$$

CASE 1 . Suppose that there exists $n_{0} \in \mathbb{N}$ such that $\left\{\left\|x_{n}-x^{*}\right\|\right\}_{n=1}^{\infty}$ is decreasing for $n \geq n_{0}$. Then the limit of $\left\{\left\|x_{n}-x^{*}\right\|\right\}_{n=1}^{\infty}$ exists. Consequently,

$$
\lim _{n \rightarrow \infty}\left(\left\|x_{n}-x^{*}\right\|^{2}-\left\|x_{n+1}-x^{*}\right\|^{2}\right)=0
$$

Moreover, by using $0<\underline{\rho} \leq \rho_{n} \leq \bar{\rho}<\min \left\{\frac{1}{2 L_{1}}, \frac{1}{2 L_{2}}\right\}$ and (12), we have

$$
\begin{aligned}
0 \leq & \left(1-\delta_{n}\right)\left(\left(1-2 \bar{\rho} L_{1}\right)\left\|x_{n}-y_{n}\right\|^{2}+\left(1-2 \bar{\rho} L_{2}\right)\left\|y_{n}-z_{n}\right\|^{2}\right) \\
& +\delta_{n}\left\|x_{n}-x^{*}\right\|^{2}-2 \delta_{n}\left\langle u-x^{*}, x_{n+1}-x^{*}\right\rangle \\
\leq & \left\|x_{n}-x^{*}\right\|^{2}-\left\|x_{n+1}-x^{*}\right\|^{2} .
\end{aligned}
$$

Since $\lim _{n \rightarrow \infty} \delta_{n}=0,\left(1-2 \bar{\rho} L_{1}\right)>0$, and $\left(1-2 \bar{\rho} L_{2}\right)>0$, then it follows from (14) and (13) that

$$
\lim _{n \rightarrow \infty}\left\|x_{n}-y_{n}\right\|=0 \quad \text { and } \quad \lim _{n \rightarrow \infty}\left\|y_{n}-z_{n}\right\|=0 .
$$

This implies that

$$
\lim _{n \rightarrow \infty}\left\|z_{n}-x_{n}\right\|=0 .
$$

By combining (10) and (11), we obtain

$$
\begin{aligned}
0 & \leq\left\|x_{n}-x^{*}\right\|^{2}-\left\|\pi_{n}-x^{*}\right\|^{2}-\delta_{n}\left\|x_{n}-x^{*}\right\|^{2}-2 \delta_{n}\left\langle u-x^{*}, x_{n+1}-x^{*}\right\rangle \\
& \leq\left\|x_{n}-x^{*}\right\|^{2}-\left\|x_{n+1}-x^{*}\right\|^{2} .
\end{aligned}
$$

Thus, from (13) and (17), we get

$$
\lim _{n \rightarrow \infty}\left(\left\|x_{n}-x^{*}\right\|^{2}-\left\|\pi_{n}-x^{*}\right\|^{2}\right)=0 .
$$

Owing to (C1), (8), and (10), we have

$$
0 \leq t_{1}\left(2-\sigma_{n}\right) F\left(z_{n}\right) \leq\left\|x_{n}-x^{*}\right\|^{2}-\left\|\pi_{n}-x^{*}\right\|^{2} .
$$

Clearly, from (19), (18), and (C2), we obtain

$$
\lim _{n \rightarrow \infty} F\left(z_{n}\right)=0 \text {. Hence } \lim _{n \rightarrow \infty}\left\|A z_{n}-u_{n}\right\|=0 .
$$


Since $\left\|A z_{n}-A x^{*}\right\| \leq\left\|A z_{n}-u_{n}\right\|+\left\|u_{n}-A x^{*}\right\|$, then it follows from (20) that

$$
\lim _{n \rightarrow \infty}\left(\left\|A z_{n}-A x^{*}\right\|^{2}-\left\|u_{n}-A x^{*}\right\|^{2}\right)=0
$$

By Lemma 3.2, we have

$$
\left\|u_{n}-A x^{*}\right\|^{2} \leq\left\|\hat{v_{n}}-A x^{*}\right\|^{2}-\left(1-2 r_{n} L_{1}\right)\left\|\hat{v_{n}}-v_{n}\right\|^{2}-\left(1-2 r_{n} L_{2}\right)\left\|v_{n}-u_{n}\right\|^{2}
$$

Similarly, from $0<\underline{r} \leq r_{n} \leq \bar{r}<\min \left\{\frac{1}{2 L_{1}}, \frac{1}{2 L_{2}}\right\}$ and (22), we get

$$
\left(1-2 \bar{r} L_{1}\right)\left\|\hat{v_{n}}-v_{n}\right\|^{2}+\left(1-2 \bar{r} L_{2}\right)\left\|v_{n}-u_{n}\right\|^{2} \leq\left\|A z_{n}-A x^{*}\right\|^{2}-\left\|u_{n}-A x^{*}\right\|^{2} .
$$

Moreover, since $\left(1-2 \bar{r} L_{1}\right)>0$ and $\left(1-2 \bar{r} L_{2}\right)>0$, then it follows from (23) and (21) that

$$
\lim _{n \rightarrow \infty}\left\|\hat{v_{n}}-v_{n}\right\|=0 \text { and } \quad \lim _{n \rightarrow \infty}\left\|v_{n}-u_{n}\right\|=0
$$

Now, let $\zeta_{n}=z_{n}-\mu_{n} G\left(z_{n}\right)$. Then

$$
\left\|\zeta_{n}-z_{n}\right\|=\mu_{n}\left\|G\left(z_{n}\right)\right\|=\mu_{n}\left\|A^{*}\right\|\left\|A\left(z_{n}\right)-u_{n}\right\|
$$

Again, by using (C1), (20), and (25), we get

$$
\lim _{n \rightarrow \infty}\left\|\zeta_{n}-z_{n}\right\|=0
$$

Since $\left\|\pi_{n}-z_{n}\right\|^{2} \leq\left\|\zeta_{n}-z_{n}\right\|^{2}$, then it follows from (26) that

$$
\lim _{n \rightarrow \infty}\left\|\pi_{n}-z_{n}\right\|=0
$$

By the triangle inequality in conjunction with (16) and (27), we obtain

$$
\lim _{n \rightarrow \infty}\left\|\pi_{n}-x_{n}\right\|=0
$$

It is clear that

$$
\left\|x_{n+1}-\pi_{n}\right\| \leq \delta_{n}\left\|u-\pi_{n}\right\|
$$

Since $\delta_{n} \rightarrow 0$ as $n \rightarrow \infty$ and $\left\{\left\|u-\pi_{n}\right\|\right\}_{n=1}^{\infty}$ is bounded, then

$$
\lim _{n \rightarrow \infty}\left\|x_{n+1}-\pi_{n}\right\|=0
$$

Consequently, from (30) and (28), we have

$$
\lim _{n \rightarrow \infty}\left\|x_{n+1}-x_{n}\right\|=0
$$


Furthermore, since $\mathrm{H}_{1}$ is reflexive and $\left\{x_{n}\right\}_{n=1}^{\infty} \subset \mathrm{H}_{1}$ is bounded, then there exists a subsequence $\left\{x_{n_{k}}\right\}_{k=1}^{\infty}$ of $\left\{x_{n}\right\}_{n=1}^{\infty}$ such that

$$
\begin{aligned}
& x_{n_{k}} \rightarrow e^{*} \in \mathrm{H}_{1} \quad \text { as } k \rightarrow \infty \text {. Therefore, assume w.o.l.o.g. that } \\
& \limsup _{n \rightarrow \infty}\left\langle u-x^{*}, x_{n}-x^{*}\right\rangle=\lim _{k \rightarrow \infty}\left\langle u-x^{*}, x_{n_{k}}-x^{*}\right\rangle .
\end{aligned}
$$

Moreover, since $\left\{z_{n}\right\}_{n=1}^{\infty},\left\{y_{n}\right\}_{n=1}^{\infty}$, and $\left\{\pi_{n}\right\}_{n=1}^{\infty}$ are bounded, then it follows from (15), (16), (28), and (32) that

$$
z_{n_{k}} \rightarrow e^{*}, \quad y_{n_{k}} \rightarrow e^{*}, \quad \pi_{n_{k}} \rightarrow e^{*} \quad \text { as } k \rightarrow \infty .
$$

It will now be shown that the weak limit $e^{*}$ solves $\operatorname{SEP}(6)$. That is, $e^{*} \in \Omega$. Indeed, since $C$ and $Q$ are closed and convex, then

$$
C \text { and } Q \text { are weakly closed. }
$$

Also, since $\left\{x_{n}\right\}_{n=1}^{\infty} \subset C$, then it follows from (32) and (35) that $e^{*} \in C$. Note that $A$ is linear and bounded. So, from (34), we obtain $A z_{n_{k}} \rightarrow A e^{*}$ as $k \rightarrow \infty$. In view of (20) and the boundedness of $\left\{u_{n}\right\}_{n=1}^{\infty}$, we see that

$$
u_{n_{k}} \rightarrow A\left(e^{*}\right) \text { as } k \rightarrow \infty \text {. }
$$

Likewise, since $\left\{v_{n}\right\}_{n=1}^{\infty}$ and $\left\{\hat{v_{n}}\right\}_{n=1}^{\infty}$ are bounded, then, from (36) and (24), we get

$$
v_{n_{k}} \rightarrow A\left(e^{*}\right), \quad \hat{n_{n_{k}}} \rightarrow A\left(e^{*}\right) \quad \text { as } k \rightarrow \infty .
$$

Clearly, since $\left\{\hat{n_{k}}\right\}_{k=1}^{\infty} \subset Q$, then, from (35) and (37), we deduce that $A\left(e^{*}\right) \in Q$. It remains to show that $e^{*} \in \operatorname{EP}(C, f)$ and $A e^{*} \in \operatorname{EP}(Q, g)$. By Lemma 3.2, in particular, for all $k \in \mathbb{N}$, we have

$$
\rho_{n_{k}}\left(f\left(x_{n_{k}}, y\right)-f\left(x_{n_{k}}, y_{n_{k}}\right)\right) \geq\left\langle y_{n_{k}}-x_{n_{k}}, y_{n_{k}}-y\right\rangle, \quad \forall y \in C .
$$

This implies

$$
\frac{\left\langle y_{n_{k}}-x_{n_{k}}, y_{n_{k}}-y\right\rangle}{\rho_{n_{k}}} \leq f\left(x_{n_{k}}, y\right)-f\left(x_{n_{k}}, y_{n_{k}}\right), \quad \forall y \in C .
$$

However, since $\rho_{n_{k}} \geq \underline{\rho}>0$, then, by applying the Cauchy-Schwartz inequality, we see that

$$
\begin{aligned}
\left|\frac{\left\langle y_{n_{k}}-x_{n_{k}}, y_{n_{k}}-y\right\rangle}{\rho_{n_{k}}}\right| & =\frac{\left|\left\langle y_{n_{k}}-x_{n_{k}}, y_{n_{k}}-y\right\rangle\right|}{\rho_{n_{k}}} \leq \frac{\left|\left\langle y_{n_{k}}-x_{n_{k}}, y_{n_{k}}-y\right\rangle\right|}{\underline{\rho}} \\
& \leq \frac{\left\|y_{n_{k}}-x_{n_{k}}\right\|\left\|y_{n_{k}}-y\right\|}{\underline{\rho}}, \quad \forall y \in C .
\end{aligned}
$$

On the other hand, since $\left\{y_{n_{k}}\right\}_{k=1}^{\infty}$ is bounded and $\lim _{k \rightarrow \infty}\left\|y_{n_{k}}-x_{n_{k}}\right\|=0$, then $\| y_{n_{k}}-$ $x_{n_{k}}\|\| y_{n_{k}}-y \| \rightarrow 0$ as $k \rightarrow \infty$. Consequently,

$$
\lim _{k \rightarrow \infty} \frac{\left\langle y_{n_{k}}-x_{n_{k}}, y_{n_{k}}-y\right\rangle}{\rho_{n_{k}}}=0 .
$$


Since $f$ satisfies (A2), then, by passing limit as $k \rightarrow \infty$ in (38) in conjunction with (34), (32), and (40), we have

$$
\begin{aligned}
\lim _{k \rightarrow \infty} \frac{\left\langle y_{n_{k}}-x_{n_{k}}, y_{n_{k}}-y\right\rangle}{\rho_{n_{k}}} & \leq \lim _{k \rightarrow \infty}\left(f\left(x_{n_{k}}, y\right)-f\left(x_{n_{k}}, y_{n_{k}}\right)\right) \\
& =f\left(e^{*}, y\right)-f\left(e^{*}, e^{*}\right), \quad \forall y \in C .
\end{aligned}
$$

Thus $e^{*} \in \operatorname{EP}(C, f)$. Again, by Lemma 3.2, we see that

$$
r_{n_{k}}\left(g\left(\hat{n_{n_{k}}}, y\right)-g\left(\hat{n_{n_{k}}}, v_{n_{k}}\right)\right) \geq\left\langle v_{n_{k}}-\hat{v_{n_{k}}}, v_{n_{k}}-v\right\rangle, \quad \forall v \in Q .
$$

Therefore

$$
\frac{\left\langle v_{n_{k}}-\hat{v_{n_{k}}}, v_{n_{k}}-v\right\rangle}{r_{n_{k}}} \leq g\left(\hat{v_{n_{k}}}, v\right)-g\left(\hat{v_{n_{k}}}, v_{n_{k}}\right), \quad \forall v \in Q
$$

Since $r_{n_{k}} \geq \underline{r}>0$, applying the Cauchy-Schwarz inequality, we have

$$
\begin{aligned}
\left|\frac{\left\langle v_{n_{k}}-\hat{v_{n_{k}}}, v_{n_{k}}-v\right\rangle}{r_{n_{k}}}\right| & =\frac{\left|\left\langle v_{n_{k}}-\hat{v_{n_{k}}}, v_{n_{k}}-v\right\rangle\right|}{r_{n_{k}}} \leq \frac{\left|\left\langle v_{n_{k}}-\hat{v_{n_{k}}}, v_{n_{k}}-v\right\rangle\right|}{\underline{r}} \\
& \leq \frac{\left\|v_{n_{k}}-\hat{v_{n_{k}}}\right\|\left\|v_{n_{k}}-v\right\|}{\underline{r}}, \quad \forall v \in Q .
\end{aligned}
$$

Now, since $\left\{v_{n_{k}}\right\}_{k=1}^{\infty}$ is bounded and $\lim _{k \rightarrow \infty}\left\|v_{n_{k}}-\hat{v_{n_{k}}}\right\|=0$, then $\left\|v_{n_{k}}-\hat{v_{n_{k}}}\right\|\left\|v_{n_{k}}-v\right\| \rightarrow 0$ as $k \rightarrow \infty$. Hence,

$$
\lim _{k \rightarrow \infty} \frac{\left\langle v_{n_{k}}-\hat{v_{n_{k}}}, v_{n_{k}}-v\right\rangle}{r_{n_{k}}}=0 .
$$

Again since $g$ satisfies (A2), then by passing limit as $k \rightarrow \infty$ in (42) using (36), (37), and (44), we get

$$
0 \leq \lim _{k \rightarrow \infty}\left(g\left(\hat{v_{n_{k}}}, v\right)-g\left(\hat{v_{n_{k}}}, v_{n_{k}}\right)\right)=g\left(A e^{*}, v\right)-g\left(A e^{*}, A e^{*}\right), \quad \forall v \in Q
$$

Hence $e^{*} \in \Omega$. Since $\Omega$ is closed, convex, and $x^{*}=\mathrm{P}_{\Omega}(u)$, then it follows from (31), (32), (33), and Lemma 2.3 that

$$
\begin{aligned}
\limsup _{n \rightarrow \infty}\left\langle u-x^{*}, x_{n+1}-x^{*}\right\rangle & \leq \limsup _{n \rightarrow \infty}\left\langle u-x^{*}, x_{n}-x^{*}\right\rangle=\lim _{k \rightarrow \infty}\left\langle u-x^{*}, x_{n_{k}}-x^{*}\right\rangle \\
& =\left\langle u-x^{*}, e^{*}-x^{*}\right\rangle \leq 0 .
\end{aligned}
$$

Consequently, by Lemma 2.5, (11), and (46), we conclude that

$$
\lim _{n \rightarrow \infty}\left\|x_{n}-x^{*}\right\|^{2}=0
$$

CASE 2. Suppose that $\left\{\left\|x_{n}-x^{*}\right\|\right\}_{n=1}^{\infty}$ is not decreasing such that there exists a subsequence $\left\{\left\|x_{n_{l}}-x^{*}\right\|\right\}_{l=1}^{\infty}$ of $\left\{\left\|x_{n}-x^{*}\right\|\right\}_{n=1}^{\infty}$ satisfying

$$
\left\|x_{n_{l}}-x^{*}\right\|<\left\|x_{n_{l+1}}-x^{*}\right\| \quad \text { for all } l \in \mathbb{N} .
$$


In view of Lemma 2.4, there exists a nondecreasing sequence $\left\{m_{k}\right\}_{k=1}^{\infty} \subset \mathbb{N}$ such that $\lim _{k \rightarrow \infty} m_{k}=\infty$ and the following inequalities hold for all $k \in \mathbb{N}$ :

$$
\left\|x_{m_{k}}-x^{*}\right\| \leq\left\|x_{m_{k+1}}-x^{*}\right\| \text { and }\left\|x_{k}-x^{*}\right\| \leq\left\|x_{m_{k+1}}-x^{*}\right\| .
$$

Note that by discarding the repeated terms of $\left\{m_{k}\right\}_{k=1}^{\infty}$, but still denoted by $\left\{m_{k}\right\}_{k=1}^{\infty}$, we can have $\left\{x_{m_{k}}\right\}_{k=1}^{\infty}$, as a subsequence of $\left\{x_{n}\right\}_{n=1}^{\infty}$. Since $\left\{x_{m_{k}}\right\}_{k=1}^{\infty}$ is bounded, then $\lim _{n \rightarrow \infty}\left(\| x_{m_{k}}-\right.$ $\left.x^{*}\|-\| x_{m_{k+1}}-x^{*} \|\right)=0$. Then, following arguments similar to those in Case 1, we deduce that

$$
\begin{aligned}
& \lim _{k \rightarrow \infty}\left\|x_{m_{k+1}}-x_{m_{k}}\right\|=0 \text { and } \\
& \limsup _{k \rightarrow \infty}\left\langle u-x^{*}, x_{m_{k+1}}-x^{*}\right\rangle \leq 0, \quad \text { where } x^{*}=\mathrm{P}_{\Omega}(u) .
\end{aligned}
$$

It follows from (47) and (11) that

$$
\begin{aligned}
\left\|x_{m_{k+1}}-x^{*}\right\|^{2} & \leq\left(1-\delta_{m_{k}}\right)\left\|x_{m_{k}}-x^{*}\right\|^{2}+2 \delta_{m_{k}}\left\langle u-x^{*}, x_{m_{k+1}}-x^{*}\right\rangle \\
& \leq\left(1-\delta_{m_{k}}\right)\left\|x_{m_{k+1}}-x^{*}\right\|^{2}+2 \delta_{m_{k}}\left\langle u-x^{*}, x_{m_{k+1}}-x^{*}\right\rangle
\end{aligned}
$$

Clearly, by dividing through by $\delta_{m_{k}}$, we get

$$
\left\|x_{m_{k+1}}-x^{*}\right\|^{2} \leq 2\left\langle u-x^{*}, x_{m_{k+1}}-x^{*}\right\rangle \text {. }
$$

Passing limit as $k \rightarrow \infty$ in (51) using (49), we obtain

$$
\lim _{k \rightarrow \infty}\left\|x_{m_{k+1}}-x^{*}\right\|^{2}=0
$$

Consequently, from (47), we see that

$$
\lim _{k \rightarrow \infty}\left\|x_{k}-x^{*}\right\|^{2}=0
$$

Hence $x_{n} \rightarrow x^{*} \in \Omega$ in both cases and this ends the proof.

\section{Remark 1}

(1) Theorem 3.3 will take the form of the extragradient method studied by Tran et al. [23] if we set $g \equiv 0$ and $\mathrm{H}_{1} \equiv \mathrm{H}_{2}$.

(2) Theorem 3.3 coincides with the work of Lopez et al. [15], whenever $g=f \equiv 0$.

(3) The restriction on the step-size $\mu \in\left(0, \frac{1}{\|A\|^{2}}\right)$ is imposed by He [12], while the step-size $\mu \in\left(0, \frac{1}{\|A\|^{2}}\right)$ is relaxed with adaptive step-size that uses a simpler initialization sequence $\left\{\sigma_{n}\right\}_{n=1}^{\infty} \subset(0,2)$ in our work.

(4) Moreover, in the work of $\mathrm{He}$ [12], a weak convergence result was obtained for solving (6) and the strong convergence follows only through the hybrid proximal point algorithm, which is not easy to implement. In this paper, we obtain a strong convergence result for solving (6) without using the hybrid scheme.

(5) The conclusion of our Theorem 3.3 holds for pseudomonotone bifunctions, while the corresponding result by $\mathrm{He}$ [12] is restricted to monotone bifunctions. 
Table 1 Example 4.1: Comparison of Algorithm 3.1 with He's algorithm (7)

\begin{tabular}{rlllll}
\hline$m$ & \multicolumn{2}{l}{ Number of iterations $(n)$} & & \multicolumn{2}{l}{ CPU time (s) } \\
\cline { 2 - 3 } & Algorithm 3.1 & He's algorithm (7) & & Algorithm 3.1 & He's algorithm (7) \\
\hline 5 & 13 & 111 & 0.0942812 & 0.8982632 \\
10 & 23 & 197 & 0.8638912 & 2.9385731 \\
20 & 33 & 201 & 1.2636421 & 4.3829392 \\
50 & 53 & 403 & 1.5485732 & 8.0197482 \\
70 & 63 & 445 & 1.8735482 & 8.6749302 \\
100 & 57 & 501 & 2.1294532 & 9.1485839 \\
150 & 51 & 499 & 2.5394313 & 12.9829482 \\
\hline
\end{tabular}

\section{Numerical results}

In this section, a preliminary numerical test is presented to compare the convergence behavior of proposed Algorithm 3.1 with algorithm (7).

Example 4.1 Consider the Nash-Cournot equilibrium problem studied in [20, 23], where $f: C \times C \rightarrow \mathbb{R}$ is defined by

$$
f(x, y)=\langle U x+V y+c, y-x\rangle,
$$

where $c \in \mathbb{R}^{m}$ and $U, V$ are two matrices of order $m$ such that $V$ is symmetric positive semidefinite and $V-U$ is negative semidefinite with Lipschitz constants $L_{1}=L_{2}=\frac{1}{2} \| U-$ $V \|$. The matrices $U, V$ are randomly generated ${ }^{1}$ and the entries of $c$ randomly belong to $[-1,1]$. The constraint set $C \subset \mathbb{R}^{m}$ is taken as follows:

$$
C:=\left\{x \in \mathbb{R}^{m}: \sum_{i=1}^{m} x_{i} \geq-1,-10 \leq x_{i} \leq 10\right\} .
$$

Assume that $g: Q \times Q \rightarrow \mathbb{R}$ is defined by $g(x, y)=x(y-x), \forall x, y \in Q=[-1, \infty)$. Suppose that $A: \mathbb{R}^{m} \rightarrow Q$ is a linear operator defined by $A x=\langle a, x\rangle, \forall x \in \mathbb{R}^{m}$, where $a$ is a vector in $\mathbb{R}^{m}$ whose elements are randomly generated from $[1 ; m]$. Thus, $A^{*}:[-1, \infty) \rightarrow \mathbb{R}^{m}$ is of the form $A^{*} y=y . a$ for all $y \in \mathbb{R}$ and $\|A\|=\|a\|$. The starting points $x_{1} \in C$ are randomly generated in the interval $[-10,10]$, and we choose $\mu=\frac{1}{2\|a\|^{2}}, \rho_{n}=r_{n}=\frac{1}{4 L_{1}}, \delta_{n}=\frac{1}{n+2}$, and $\sigma_{n}=2-\frac{1}{n+1}$. We define the function $T O L_{n}$ by $T O L_{n}:=\left\|x_{n+1}-x_{n}\right\|$ and use the stopping rule $T O L_{n}<\epsilon$ for the iterative process, where $\epsilon$ is the predetermined error. The equivalent convex quadratic problems are solved using the function fmincon and implemented in MATLAB 7.0 running on an HP Compaq510, Core(TM)2 Duo Processor T5870 with $2.00 \mathrm{GHz}$ and $2 \mathrm{~GB}$ RAM. Table 1 shows that Algorithm 3.1 outperforms He's algorithm (7) in running time and in the number of iterations for different cases of $m$.

\section{Conclusion}

In this paper, we have proposed a self-adaptive extragradient iterative process for solving split pseudomonotone equilibrium problems. We established strong convergence of our proposed algorithm, and the performance of the algorithm such as CPU time and the

${ }^{1}$ Two matrices are randomly generated $E$ and $F$ with entries from $[-1,1]$. The matrix $V=E^{\top} E, S=F^{\top} F$ and $U=S+V$. 
number of iterations required for convergence is highlighted through preliminary numerical tests that show that our proposed algorithm is faster than the corresponding algorithm by He [12].

\section{Acknowledgements}

The authors are grateful to the editor and anonymous referees for their valuable suggestions and constructive comments which have improved this paper. Yusuf I. Suleiman is grateful to King Mongkut's University of Technology Thonburi (KMUTT), Bangkok 10140, Thailand, for providing state-of-the-art research facilities to carry out this research work during his bench work in KMUTT. The authors acknowledge the financial support provided by the Center of Excellence in Theoretical and Computational Science (TaCS-COE), KMUTT. Moreover, this research project is supported by Thailand Science Research and Innovation (TSRI) Basic Research Fund: Fiscal year 2021 under project number 64A306000005.

\section{Funding}

The authors acknowledge the financial support provided by the Center of Excellence in Theoretical and Computational Science (TaCS-CoE), KMUTT. Moreover, this research project is supported by Thailand Science Research and Innovation (TSRI) Basic Research Fund: Fiscal year 2021 under project number 64A306000005.

\section{Availability of data and materials}

Not applicable.

\section{Competing interests}

The authors declare that they have no competing interests.

\section{Authors' contributions}

All authors contributed equally and significantly in writing this article. All authors read and approved the final manuscript.

\section{Author details}

${ }^{1}$ Department of Mathematics, Kano University of Science and Technology, Wudil, Nigeria. ${ }^{2}$ Center of Excellence in Theoretical and Computational Science (TaCS-CoE) \& KMUTTFixed Point Research Laboratory, Room SCL 802 Fixed Point Laboratory, Science Laboratory Building, Departments of Mathematics, Faculty of Science, King Mongkut's University of Technology Thonburi (KMUTT), 126 Pracha-Uthit Road, Bang Mod, Thung Khru, Bangkok 10140, Thailand. ${ }^{3}$ Department of Medical Research, China Medical University Hospital, China Medical University, Taichung 40402, Taiwan. ${ }^{4}$ Applied Mathematics for Science and Engineering Research Unit (AMSERU), Program in Applied Statistics, Department of Mathematics and Computer Science, Faculty of Science and Technology, Rajamangala University of Technology Thanyaburi, Thanyaburi, Pathumthani 12110, Thailand.

\section{Publisher's Note}

Springer Nature remains neutral with regard to jurisdictional claims in published maps and institutional affiliations.

Received: 24 February 2021 Accepted: 14 July 2021 Published online: 02 August 2021

\section{References}

1. Anh, P.N.: A hybrid extragradient method extended to fixed point problems and equilibrium problems. Optimization 62, 271-283 (2013)

2. Bianchi, M., Schaible, S.: Generalized monotone bifunctions and equilibrium problems. J. Optim. Theory Appl. 90, 31-34 (1996)

3. Bryne, C.: A unified treatment of some iterative algorithms in signal processing and image reconstruction. Inverse Probl. 20, 103-120 (2004)

4. Censor, Y., Bortfeld, T., Martin, B., Trofimov, A.: A unified approach for inversion problems in intesity-modulated radiation therapy. Phys. Med. Biol. 51, 2353-2365 (2006)

5. Censor, Y., Elfving, T.: A multiprojection algorithm using Bregman projection in a product space. Numer. Algorithms 8 , 221-239 (1994)

6. Censor, Y., Elfving, T., Kopf, N., Bortfed, T.: The multiple-set split feasibility problem and its applications for inverse problems. Inverse Probl. 21, 2071-2084 (2005)

7. Censor, Y., Gibali, A., Reich, S.: Algorithms for the split variational inequality problem. Numer. Algorithms 59, 301-323 (2012)

8. Combettes, P.L., Hirstoaga, S.A.: Equilibrium programming in Hilbert spaces. J. Nonlinear Convex Anal. 6, 117-136 (2005)

9. Dang, V.H.: Convergence analysis of a new algorithm for strongly pseudomontone equilibrium problems. Numer. Algorithms 77, 983-1001 (2018)

10. Fan, K.: A minimax inequality and applications. In: Inequalities III, pp. 103-113. Academic Press, New York (1972)

11. He, B., He, X., Liu, H., Wu, T.: Self-adaptive projection method for co-coercive variational inequalities. Eur. J. Oper. Res. $196,43-48(2009)$

12. He, Z.: The split equilibrium problem and its convergence algorithms. J. Inequal. Appl. 2012, Article ID 162 (2012)

13. Kim, J.K., Majee, P.: Modified Krasnoselski-Mann iterative method for hierarchical fixed point problem and split mixed equilibrium problem. J. Inequal. Appl. 2020, Article ID 227 (2020)

14. Kim, J.K., Salahuddin, S.: Existence of solutions for multivalued equilibrium problems. Nonlinear Funct. Anal. Appl. 23(4), 779-795 (2018) 
15. Lopez, G., Martin-Marquez, V., Wang, F.H., Xu, H.K.: Solving the split feasibility problem without prior knowledge of matrix norms. Inverse Probl. 28, Article ID 085004 (2012)

16. Mainge, P.E.: Strong convergence of projected subgradient methods for nonsmooth and nonstrictly convex minimization. Set-Valued Anal. 16, 899-912 (2008)

17. Moudafi, A.: Split monotone variational inclusions. J. Optim. Theory Appl. 50, 275-283 (2011)

18. Oyewole, O.K., Mewomo, O.T.: Existence results for new generalized mixed equilibrium and fixed point problems in Banach spaces. Nonlinear Funct. Anal. Appl. 25(2), 273-301 (2020)

19. Rehman, H.U., Kumam, P., Cho, Y.J., Suleiman, Y.I., Kumam, W.: Modified Popov's explicit iterative algorithms for solving pseudomonotone equilibrium problems. Optim. Methods Softw. (2020). https://doi.org/10.1080/10556788.2020.1734805

20. Suleiman, Y.I., Rehman, H.U., Gibali, A., Kumam, P.: A self-adaptive extragradient CQ-method for a class of bilevel split equilibrium problem with application to Nash Cournot oligopolistic electricity market models. Comput. Appl. Math. $39,293(2020)$

21. Tam, N.N., Yao, J.C., Yen, N.D.: Solution methods for pseudomonotone variational inequalities. J. Optim. Theory Appl. $138,253-273(2008)$

22. Tang, Y., Gibali, A.: New self-adaptive step size algorithms for solving split variational inclusion problems and its applications. Numer. Algorithms 83, 305-331 (2020)

23. Tran, D.Q., Dung, L.M., Nguyen, V.H.: Extragradient algorithms extended to equilibrium problems. Optimization 57, 749-776 (2008)

24. Wang, S.H., Liu, X.M., An, Y.S.: A new iterative algorithm for generalized split equilibrium problem in Hilbert spaces. Nonlinear Funct. Anal. Appl. 22(4), 911-924 (2017)

25. Xu, H.K.: An iterative approach to quadratic optimization. J. Optim. Theory Appl. 116, 659-678 (2003)

26. Yang, Q.: On variable-step relaxed projection algorithm for variational inequalities. J. Math. Anal. Appl. 302, 166-179 (2005)

27. Yao, Y., Yao, Z., Abdou, A.N., Cho, Y.J.: Self-adaptive algorithms for proximal split feasibility problems and strong convergence analysis. Fixed Point Theory Appl. 2015, 205 (2015)

28. Zhang, W., Han, D., Li, Z.: A self-adaptive projection method for solving the multiple-sets split feasibility problem. Inverse Probl. 25, 115001 (2009)

\section{Submit your manuscript to a SpringerOpen ${ }^{\circ}$ journal and benefit from:}

- Convenient online submission

- Rigorous peer review

- Open access: articles freely available online

- High visibility within the field

- Retaining the copyright to your article

Submit your next manuscript at $\gg$ springeropen.com 